\title{
Dominancia Vascular en la Mano: Estudio Dinámico de Flujos
}

\section{Arterial Dominance for Blood Supply in the Hand: Dynamic Blood Flow Study}

\author{
Carlos Eduardo Torres Fuentes ${ }^{1}$ Ana María Sastre Zuluaga ${ }^{2}$ \\ ${ }^{1}$ Especialista de Cirugía Plástica, Reconstructiva y Estética, Cirugía de \\ la mano y Microcirugía, Hospital de San José, Instructor asociado - \\ Fundación Universitaria de Ciencias de la Salud, Bogotá, Colombia \\ ${ }^{2}$ Especialista en Cirugía Plástica, Estética y Reconstructiva, Hospital \\ de San José, Fundación Universitaria de Ciencias de la Salud, Bogotá, \\ Address for correspondence Carlos Eduardo Torres Fuentes, MD, \\ Especialista de Cirugía Plástica, Reconstructiva y Estética, Cirugía de la \\ mano y Microcirugía, Hospital de San José, Instructor asociado - \\ Fundación Universitaria de Ciencias de la Salud, Bogotá, Colombia \\ (e-mail: drtorresplastic@yahoo.com).
} Colombia

Rev Iberam Cir Mano 2017;45:28-34.

\begin{abstract}
Resumen
Introducción Mediante estudios anatómicos estáticos, se ha establecido durante años, que la arteria cubital es dominante para la irrigación de la mano en un $80 \%$ de los casos. Sin embargo, estudios anatómico-radiológicos más recientes, sobre muestras poblacionales limitadas ( $n=27)$, ponen en duda la dominancia arterial cubital única de la mano. Por otro lado, el comportamiento funcional adaptativo, tanto de la arteria radial como de la cubital, cuando la otra arteria es obstruida, todavía no ha sido estudiado. Los objetivos del presente trabajo son: (1) Determinar si existe una dominancia arterial en la mano y (2), estudiar las variaciones del flujo que experimentan tanto la arteria radial como la cubital de voluntarios sanos, cuando la otra arteria se obstruye durante la realización de la prueba de Allen.

Material y Método Se realizó un estudio observacional descriptivo transversal, sobre 200 manos de 100 voluntarios sanos. Se recogieron los datos de sexo y lateralidad. Se utilizó un ecógrafo doppler color para determinar, antes y durante la realización de un test de Allen, el diámetro transversal, el área de sección, la tasa y la velocidad de flujo, tanto de la arteria radial como de la arteria cubital. Los datos recogidos se compararon con una prueba de la suma de rangos de Wilcoxon y se realizó un análisis de correlación mediante la prueba $\mathrm{P}$ de Pearson entre las diferentes variables.

Resultados No se observaron diferencias significativas entre los diferentes paráme-

Palabras Clave

- mano

- arteria cubital

- arteria radial

- ultrasonografía doppler

- colgajo tros estudiados comparándolos según la lateralidad y el género de los voluntarios evaluados. Se evidenció una diferencia estadísticamente significativa para la tasa de flujo, área de sección y diámetro transversal entre la arteria radial y cubital, tanto en reposo como durante la prueba de Allen, siendo mayor los parámetros para la arteria radial. Durante la realización de la prueba de Allen, se obtuvo una correlación positiva directa entre el incremento en el diámetro, tanto de la arteria radial como de la cubital, y de sus velocidades de flujo; se observó, un aumento en el área de sección y en la tasa de flujo de ambas al ocluir la otra.
\end{abstract}

received

March 23, 2017

accepted

March 27, 2017
DOI https://doi.org/

$10.1055 / \mathrm{s}-0037-1602794$ ISSN 1698-8396.
Copyright $\odot 2017$ Thieme Revinter

Publicações Ltda, Rio de Janeiro, Brazil
License terms

c) $(1) \$$ 
Conclusiones Ha quedado evidenciado, por medio de este estudio dinámico de flujo, una dominancia de la arteria radial para la irrigación de la mano. También ha podido demostrarse que existen cambios adaptativos, anatómicos y funcionales, en la arteria cubital y radial, cuando hay ausencia de la otra.

\section{Abstract}

\section{Keywords}

- hand

- ulnar artery

- radial artery

- doppler ultrasonography

- flap
Purpose Through some static anatomical studies, it has been established for years that the ulnar artery is dominant for the hand irrigation in $80 \%$ of cases. However, more recent anatomical and radiological studies, undertaken on limited population samples ( $n=27)$, question the unique ulnar arterial dominance of the hand. On the other hand, the adaptive functional behavior of both, the radial and the ulnar artery, when the other artery is obstructed, has not yet been studied. The purposes of this paper are: (1) To determine whether there is an arterial dominance for blood supply in the hand and (2) to study the variations in the blood flow experienced by both, the radial and the ulnar arteries of healthy volunteers, when the other artery is obstructed during the performance of an Allen test.

Methods An observational cross-sectional study was performed on 200 hands of 100 healthy volunteers. Data was collected on sex and laterality of the dominant hand. A color Doppler ultrasound was used to determine, before and during the performance of the Allen test, the transverse diameter, the cross-sectional area, the rate and the blood flow velocity, both, in the radial and the ulnar artery. The collected data was compared with a Wilcoxon signed-rank test and Pearson correlation test was used.

Results There were no significant differences between the different parameters studied comparing them according to the hand laterality and the gender of the evaluated volunteers. The radial artery blood flow rate, cross-sectional area and transvers diameter data were statistically significantly higher than the ulnar artery's, both, at rest and during the Allen test performance. During the Allen test performance, a direct positive correlation was obtained between the increase in the diameter of both, the radial and the ulnar artery, and their blood flow velocities; an increase in the crosssectional area and in the blood flow rate was observed in both arteries when the other one was ocludded.

Conclusions This dynamic blood flow study has evidenced a radial artery dominance of the hand. It has also demonstrated that there are some adaptive changes, both anatomical and functional, in both, the ulnar and the radial arteries, when there is an absence of the other one. This represents a dynamic system able to adapt to any present scenario.

\section{Introducción}

La irrigación de la mano se produce principalmente por la arteria cubital y la arteria radial tras su origen a partir de la arteria braquial en el antebrazo.A través de estudios anatómicos estáticos, se evidenció que el arco palmar superficial, formado por la arteria cubital, está presente en un 37\% de las manos y que en un $13 \%$ la arteria radial termina a nivel de la muñeca ${ }^{1}$, por lo que se ha planteado durante años, que la arteria cubital es dominante sobre la arteria radial en un $80 \%$ de la población. Por ello, de manera tradicional, en el momento de enfrentarse a una lesión de la arteria cubital, se ha establecido la necesidad de una pronta revasculariza- ción, y se ha recomendado utilizar para el diseño y disección de colgajos libres o convencionales, de manera exclusiva, la arteria radial. ${ }^{2,3}$

Con frecuencia, se han descrito complicaciones importantes al emplear la arteria radial, sin embargo se ha hecho un uso limitado de la arteria cubital en cirugía reconstructiva. ${ }^{4}$ Muchos autores han investigado la dominancia real de la vascularización de la mano por medio de diferentes métodos clínicos y de imágen, que incluyen la Prueba de Allen tradicional, la Prueba de Allen objetiva utilizando Ecografía Doppler color, ${ }^{5}$ uso de radioisótopos, ${ }^{6}$ la pletismografía de 5 canales, al igual que estudios 
morfológicos en cadáveres para lograr mediciones de los diámetros arteriales en la muñeca y en la mano. ${ }^{2}$

Se ha logrado demostrar que puede existir una dominancia de la arteria radial sobre la arteria cubital para la irrigación de la mano o incluso no existir una diferencia anatómica significativa entre las dos. ${ }^{7}$ Sin embargo, no se ha evaluado aún el comportamiento de cada uno de los vasos arteriales mediante la oclusión del otro.

La prueba de Allen fue descrita por el doctor Edgar Allen en 1929, como un método para diagnosticar enfermedad oclusiva de la arteria cubital en tres pacientes con tromboangitis obliterans, realizando compresión manual de las dos arterias y posteriormente liberando una de ellas para evaluar la suplencia vascular de cada uno de los dos vasos para la mano, estando el vaso contrario ocluido. ${ }^{8}$ Esa prueba sigue siendo hasta el día de hoy, una de las pruebas clínicas más utilizadas por especialistas para valorar la perfusión de la mano previamente a la disección y sección de la arteria radial o cubital, con el objetivo de ser utilizadas en procedimientos reconstructivos. ${ }^{8} \mathrm{Al}$ utilizar la prueba de Allen al tiempo con pruebas vasculares no invasivas como la ecografía doppler color, con la cual se puede evaluar el flujo de la arteria por la presencia de ondas de pulsación y señal de color, se puede evaluar el diámetro de la arteria y la velocidad de flujo en el monitor. ${ }^{9,10}$ Teniendo en cuenta su bajo costo y disponibilidad intrahospitalaria en servicios de radiología ${ }^{11}$, ese procedimiento puede constituir el método inicial de evaluación en algunos pacientes previo al diseño de un colgajo libre o convencional, y se pueden igualmente emplear para monitorizar al paciente de manera pre, intra o postoperatoria. ${ }^{9,10}$

El objetivo de este estudio fue establecer de manera no invasiva, mediante la aplicación de esas dos pruebas, cuál es la capacidad de adaptación de las arterias radial y cubital ante la obstrucción o ausencia de uno u otro vaso, y si realmente existe una dominancia vascular en la mano, de individuos sanos; libres de enfermedad vascular, enfermedades autoinmunes o con antecedente de procedimientos quirúrgicos realizados en los antebrazos. ${ }^{11}$

\section{Material y Método}

Se realizó un estudio observacional descriptivo de corte transversal, en el que se incluyeron cien voluntarios sanos entre 1865 años. Se realizó un análisis de acuerdo a la lateralidad y de acuerdo a la arteria analizada (cubital o radial), en usuarios del servicio de radiología y cirugía plástica del hospital de San José y del Hospital Infantil Universitario de San José, así como estudiantes, residentes y docentes de la Fundación Universitaria de Ciencias de la Salud-FUCS que formaran parte del servicio de cirugía plástica y radiología. Se excluyeron aquellos con antecedentes de enfermedad vascular obstructiva crónica, enfermedades autoinmunes o que hubieran sido sometidos a procedimientos quirúrgicos en antebrazos.

Para la realización del estudio se obtuvo la autorización del Comité de Ética e Investigación en Seres Humanos (CEISH) de la Fundación Universitaria de Ciencias de la Salud-FUCS, como también la firma del consentimiento informado por parte de todos los participantes.
Tabla 1 Características sociodemográficas de la población

\begin{tabular}{|l|l|}
\hline & TOTAL $(\boldsymbol{n}=\mathbf{1 0 0})$ \\
\hline Edad Media (RIQ) años & $32(17-86)$ \\
\hline Sexo \% hombres & 55 \\
\hline Lateralidad \% derecha & 96 \\
\hline
\end{tabular}

Abreviaciones: Med, mediana; RIQ, rango intercuartil.

La población del estudio incluyó 100 voluntarios, reclutados entre junio y septiembre del año 2014, de los cuales el $55 \%$ eran hombres, con una mediana de edad de 32 años (RIQ 27-36), y una clara predominancia de la lateralidad diestra (96\%), en quienes se obtuvieron registros de ecografía doppler color de las arterias radial y cubital de manera bilateral, en reposo y durante la prueba de Allen. (Tabla 1)

Se evaluó en los sujetos de estudio, por medio de ecografía doppler color, el comportamiento de las arterias radial y cubital en reposo y durante la oclusión de una $\mathrm{u}$ otra mediante la prueba de Allen, registrando el diámetro transversal, $(\mathrm{mm})$, área de sección $\left(\mathrm{mm}^{2}\right)$, tasa de flujo $(\mathrm{cm} / \mathrm{seg})$ y velocidad de flujo ( $\mathrm{cm} / \mathrm{seg}$ ). El personal encargado fue entrenado en ecografía doppler color y en realizar la prueba de Allen bajo los mismos parámetros en todos los voluntarios evaluados. Se determinó como dominante al vaso con valores mayores estadísticamente significativos para tres de los cuatro parámetros evaluados.

Para eso, se utilizó un ecógrafo de marca Toshiba, línea Xario, de última generación, equipado con transductores de alta resolución (mayor a $10 \mathrm{MHz}$ ) y con parámetros para estudio de doppler color y espectral, así como doppler de poder (-Figs. 1-4).

La información obtenida fue tabulada en una base de datos de Excel ${ }^{\circledR}$, posteriormente los datos fueron analizados con el software Stata $13 \AA$ el análisis descriptivo de la información se reportó con medidas de tendencia central y de dispersión para las variables consideradas; además, se realizó un análisis de correlación mediante la prueba $\mathrm{P}$ de Pearson entre las diferentes variables. Se realizó una comparación de las medianas de las arterias cubital y radial en reposo y durante la oclusión de una u otra mediante la prueba de Allen, comparando el diámetro transversal, el área de sección, la tasa de flujo y la velocidad de flujo con la prueba de suma de rangos Wilcoxon, considerándose estadísticamente significativas cuando se obtuvo un valor $p<0.05$.

\section{Resultados}

La mediana de los diámetros transversos obtenidos durante el reposo para las arterias cubitales derechas fueron de $2,3 \mathrm{~mm}$ (RIQ 2-2,5) y en el lado izquierdo 2,25 mm (RIQ $2-2,4)$, durante el reposo. Durante la prueba de Allen fueron de $2,5 \mathrm{~mm}$ (RIQ 2,25-2,8) y 2,4 (RIQ 2,2-2,7) respectivamente. Para las arterias radiales se encontraron medianas de $2,6 \mathrm{~mm}(2,3-3,05)$ y $2,7 \mathrm{~mm}(2,3-3,05)$, para derecha e izquierda durante el reposo y de $2,95^{2,3,5}$ y $3 \mathrm{~mm}(2,5-$ $3,35)$ durante la prueba clínica, respectivamente. Además 


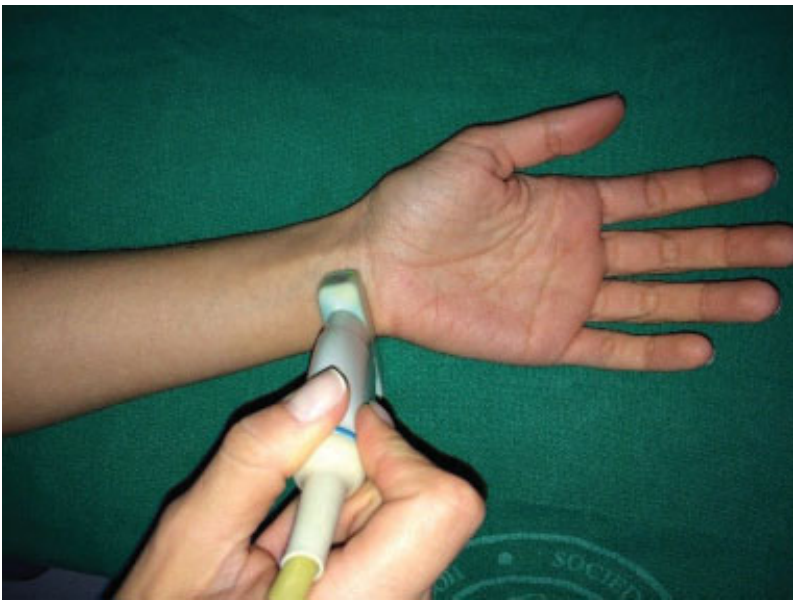

Fig. 1 Medición de flujo para comparación de las medianas de un vaso en reposo.

se calcularon las medianas para cada uno de los parámetros independientemente de la lateralidad. (Tabla 2)

Las medianas de diámetro transverso y velocidad de flujo, en reposo, para la mano izquierda, en la arteria cubital fueron de $2,25 \mathrm{~mm}$ y $34,8 \mathrm{~cm} / \mathrm{seg}$, respectivamente; mientras que para la arteria radial fueron de $2,7 \mathrm{~mm}$ y $37,3 \mathrm{~cm} /$ seg. (Tabla 3)

Durante la prueba de Allen se obtuvieron registros en la arteria cubital de $2,4 \mathrm{~mm}$ y $44,6 \mathrm{~cm} / \mathrm{seg}$, y en la arteria radial diámetro transverso de $3 \mathrm{~mm}$ y velocidad de flujo 49,1, con valores por encima de 0.8 en la prueba P de Pearson.

Se encontró correlación positiva entre el diámetro de las estructuras vasculares, y las velocidades de flujo, durante la realización de las pruebas de Allen, y por consiguiente se presentó aumento en el área de sección y en la tasa de flujo al ser dependientes del diámetro transversal y de la velocidad respectivamente.

Cuando se realizó la comparación de medianas en el caso del diámetro transversal, área de sección y tasa de flujo, ellas fueron estadísticamente significativas $p<0.05$, siendo

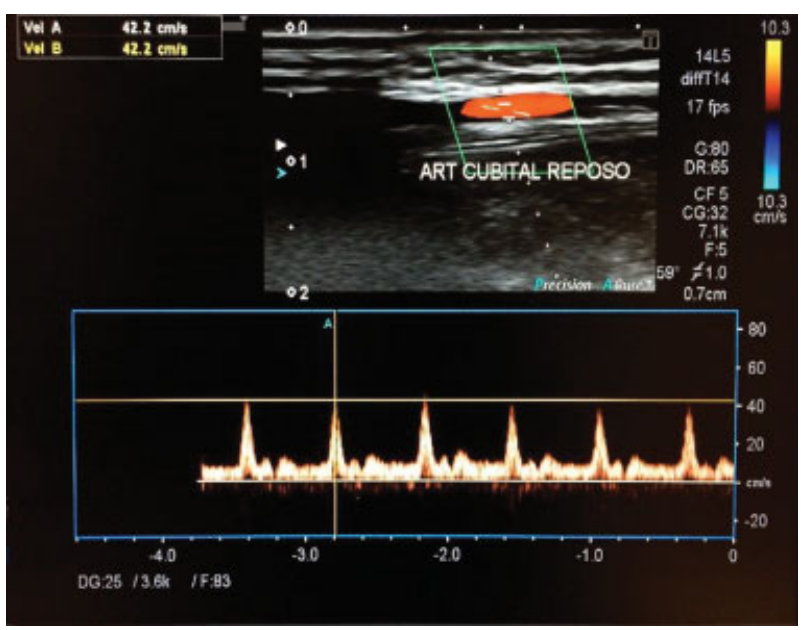

Fig. 2 Velocidad de flujo para comparación de las medianas de un vaso en reposo.

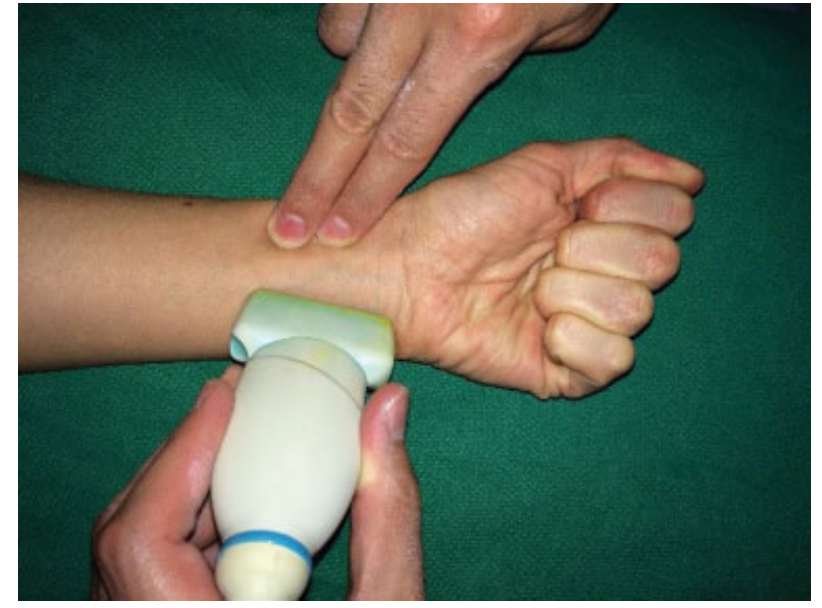

Fig. 3 Medición de flujo para comparación de las medianas de un vaso durante la prueba de Allen.

mayores los valores para la arteria radial durante la prueba de Allen. Sin embargo, cuando se compararon las medianas de velocidad de flujo, éstas no fueron estadísticamente significativas con un valor $\mathrm{P}>0.05$ entre un vaso y otro en reposo o durante la prueba de Allen (-Figs. 5-8).

Se encontraron coeficientes de correlación positivos para las velocidades de flujo y el diámetro transverso en reposo y con la prueba de Allen, con valores entre 0,8 y 0,7 para las arterias radial y cubital, de manera bilateral en la prueba $P$ de Pearson. Pearson (-Figs. 9-12).

\section{Discusión}

La irrigación arterial de la mano ha sido estudiada durante años, siendo considerado como vaso dominante la arteria cubital por hallazgos en disecciones anatómicas. ${ }^{7}$ Estudios previos han buscado verificar si realmente existe dominancia entre las dos arterias para la irrigación de la mano mediante diferentes métodos dinámicos que incluyen estudios ecográficos y el uso de doppler color en pacientes sanos, pero con muestras de sólo 27 participantes. ${ }^{9}$

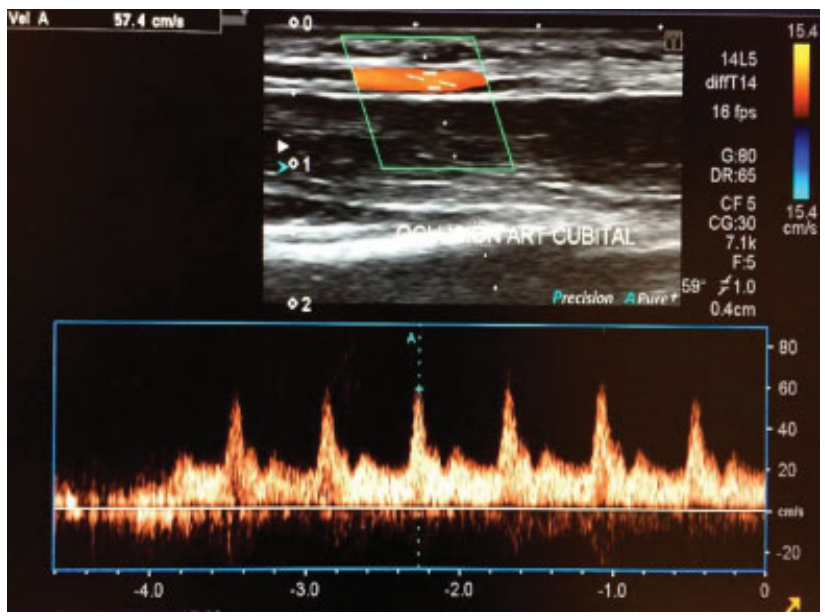

Fig. 4 Velocidad de flujo para comparación de las medianas de un vaso durante la prueba de Allen. 
32 Dominancia Vascular en la Mano: Estudio Dinámico de Flujos Fuentes, Sastre

Tabla 2 Cálculo de las medianas para cada uno de los parámetros discriminando lateralidad

\begin{tabular}{|c|c|c|c|c|c|}
\hline & & \multicolumn{2}{|l|}{ Derecha } & \multicolumn{2}{|l|}{ Izquierda } \\
\hline & & $\begin{array}{l}\text { Cubital Med } \\
\text { (RIQ) }\end{array}$ & $\begin{array}{l}\text { Radial Med } \\
\text { (RIQ) }\end{array}$ & $\begin{array}{l}\text { Cubital } \\
\text { Med (RIQ) }\end{array}$ & $\begin{array}{l}\text { Radial Med } \\
\text { (RIQ) }\end{array}$ \\
\hline \multirow[t]{4}{*}{ Reposo } & Diam. Transverso (mm) & $\begin{array}{l}2.3 \\
(2-2.5)\end{array}$ & $\begin{array}{l}2.6 \\
(2.3-3)\end{array}$ & $\begin{array}{l}2.2 \\
(2.3-3)\end{array}$ & $\begin{array}{l}2.7 \\
(2.3-3)\end{array}$ \\
\hline & Área de sección $\left(\mathrm{mm}^{2}\right)$ & $\begin{array}{l}4.15 \\
(3.1-4.9)\end{array}$ & $\begin{array}{l}5.3 \\
(4.2-7.3)\end{array}$ & $\begin{array}{l}3.9 \\
(3.1-4.5)\end{array}$ & $\begin{array}{l}5.7 \\
(4.2-7.3)\end{array}$ \\
\hline & Tasa de flujo $(\mathrm{cm} / \mathrm{seg})$ & $\begin{array}{l}1.6 \\
(1.1-2.2)\end{array}$ & $\begin{array}{l}2 \\
(1.3-3)\end{array}$ & $\begin{array}{l}1.3 \\
(1-1.9)\end{array}$ & $\begin{array}{l}2.1 \\
(1.3-2.9)\end{array}$ \\
\hline & Vel. de flujo (cm/seg) & $\begin{array}{l}41.1 \\
(29.6-48.5)\end{array}$ & $\begin{array}{l}38.2 \\
(32.3-44.5)\end{array}$ & $\begin{array}{l}34.8 \\
(29.1-41.6)\end{array}$ & $\begin{array}{l}37.3 \\
(29.9-43.6)\end{array}$ \\
\hline \multirow[t]{4}{*}{ Prueba de Allen } & Diam. Transverso (mm) & $\begin{array}{l}2.5 \\
(2.25-2.8)\end{array}$ & $\begin{array}{l}2.9 \\
(2.5-3.3)\end{array}$ & $\begin{array}{l}2.4 \\
(2.2-2.7)\end{array}$ & $\begin{array}{l}3 \\
(2.5-3.3)\end{array}$ \\
\hline & Área de sección $\left(\mathrm{mm}^{2}\right)$ & $\begin{array}{l}4.9 \\
(4-6.2) \\
\end{array}$ & $\begin{array}{l}6.8 \\
(4.9-8.6)\end{array}$ & $\begin{array}{l}4.5 \\
(3.8-5.7)\end{array}$ & $\begin{array}{l}7.1 \\
(4.9-8.8)\end{array}$ \\
\hline & Tasa de flujo $(\mathrm{cm} / \mathrm{seg})$ & $\begin{array}{l}2.5 \\
(1.9-3.4)\end{array}$ & $\begin{array}{l}3.2 \\
(1.9-4.9)\end{array}$ & $\begin{array}{l}2.1 \\
(1.5-2.9)\end{array}$ & $\begin{array}{l}3.6 \\
(2-4.6)\end{array}$ \\
\hline & Vel. de flujo $(\mathrm{cm} / \mathrm{seg})$ & $\begin{array}{l}51 \\
(43.1-63.4)\end{array}$ & $\begin{array}{l}49.8 \\
(37.9-62.2)\end{array}$ & $\begin{array}{l}44.6 \\
(39.3-56.2)\end{array}$ & $\begin{array}{l}49.1 \\
(31.3-57.1)\end{array}$ \\
\hline
\end{tabular}

Abreviaciones: Diam., diámetro; Med, mediana; RIQ, rango intercuartil; seg, segundos; Vel., velocidad.

Sin embargo, no se encontró en la literatura, un estudio que involucre condiciones clínicas similares a obstrucción o ausencia de una de las arterias principales y los cambios que se puedan observar como respuesta a esas condiciones, como lo propuesto en este estudio. ${ }^{11}$ Tampoco se encontraron estudios con una muestra de tamaño similar a la obtenida para esta investigación, con un total de cuatrocientas arterias evaluadas. ${ }^{11}$

La respuesta de las estructuras arteriales evaluadas de la mano, demostró un aumento en todos los parámetros, tanto anatómicos, como funcionales, cuando se crea un escenario similar a la obstrucción, lesión o ausencia quirúrgica de las arterias cubital o radial, para los dos vasos evaluados.

Los cambios observados en el diámetro transversal de todas las estructuras vasculares, sean o no dominantes, indican su capacidad adaptativa en cuanto al componente estructural, tanto en reposo como durante la prueba clínica. Eso fue acompañado de aumentos significativos en las velocidades de flujo y las tasas de flujo durante la recreación de condiciones obstructivas al realizar la prueba de Allen, lo que indica una capacidad adaptativa también funcional, con los cambios hemodinámicos favorables para asegurar la irrigación arterial en la mano.

Como hallazgo importante, se evidenciaron diferencias estadísticamente significativas de la tasa de flujo, área de sección y diámetro transversal entre los dos vasos, siendo los valores más elevados para la arteria radial, lo que indicaría una dominancia de ésa sobre la arteria cubital, refutando lo antiguamente estipulado para la irrigación de la mano.

La disponibilidad, bajo costo y reproducibilidad del uso de ecografía doppler color lo convierten en el medio de elección adecuado para una valoración de la irrigación arterial, previo

Tabla 3 Cálculo de las medianas para cada uno de los parámetros independientemente de la lateralidad

\begin{tabular}{|l|l|l|l|}
\hline Variable & & $\begin{array}{l}\text { Arteria Cubital } \boldsymbol{n}=\mathbf{2 0 0} \\
\text { Med (RIQ) }\end{array}$ & Arteria Radial $\mathbf{n}=\mathbf{2 0 0}$ Med (RIQ) \\
\hline \multirow{4}{*}{ Reposo } & Diam. Transverso $(\mathrm{mm})$ & $2.3(2.0-2.4)$ & $2.65(2.3-3.05)$ \\
\cline { 2 - 4 } & Área de sección $\left(\mathrm{mm}^{2}\right)$ & $4.1(3.1-4.5)$ & $5.5(4.1-7.3)$ \\
\cline { 2 - 4 } & Tasa de flujo $(\mathrm{cm} / \mathrm{seg})$ & $1.44(1.0-1.9)$ & $2.11(1.3-2.9)$ \\
\cline { 2 - 4 } & Vel. de flujo $(\mathrm{cm} / \mathrm{seg})$ & $37.3(29.2-47)$ & $37.4(31.3-44.1)$ \\
\hline Prueba de Allen & Diam. Transverso $(\mathrm{mm})$ & $2.4(2.2-2.7)$ & $3.0(2.5-3.3)$ \\
\cline { 2 - 4 } & Área de sección $\left.(\mathrm{mm})^{2}\right)$ & $4.52(3.8-5.7)$ & $3.07(4.9-8.5)$ \\
\cline { 2 - 4 } & Tasa de flujo $(\mathrm{cm} / \mathrm{seg})$ & $2.28(1.6-3.2)$ & $49.2(38.5-59.3)$ \\
\cline { 2 - 4 } & Vel. de flujo $(\mathrm{cm} / \mathrm{seg})$ & $49.25(40-60)$ & $4.7)$ \\
\hline
\end{tabular}

Abreviaciones: Diam., diámetro; Med, mediana; RIQ, rango intercuartil; seg, segundos; Vel., velocidad. 


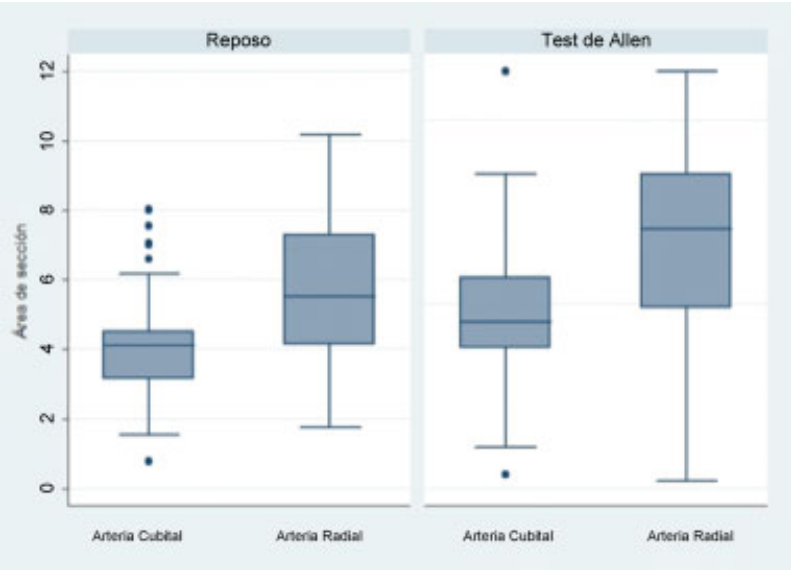

Fig. 5 Coeficientes de correlación para el área de sección en reposo y con la prueba de Allen para las arterias radial y cubital.

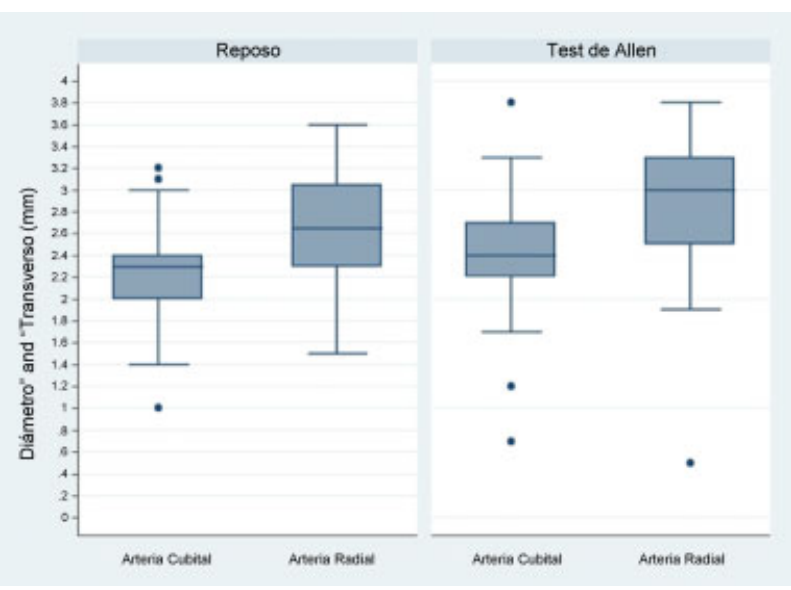

Fig. 6 Coeficientes de correlación para el diámetro transversal en reposo y con la prueba de Allen para las arterias radial y cubital.

a los procedimientos reconstructivos, con lo cual se puede escoger más acertadamente cuál de las dos arterias puede ser usada en esos eventos y se asegura la disminución de posibles complicaciones relacionadas al flujo arterial residual en el miembro afectado.

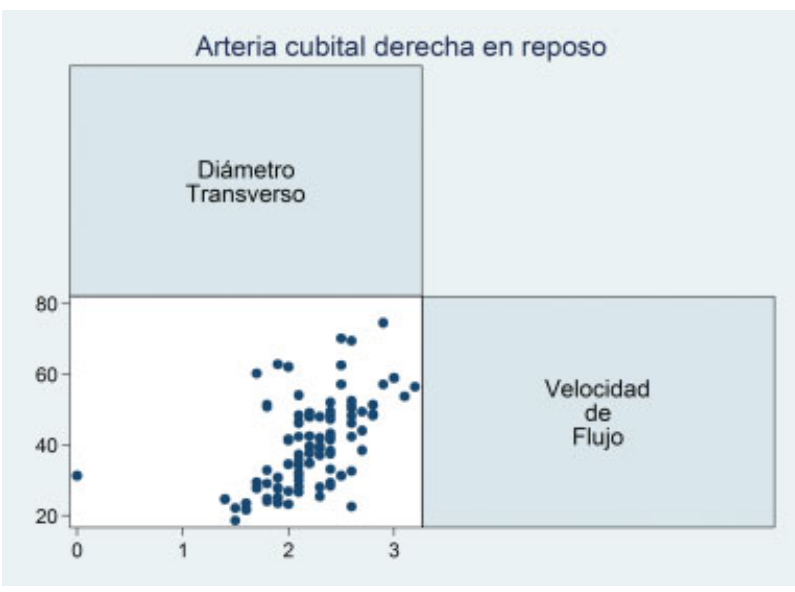

Fig. 9 Arteria cubital derecha en reposo.

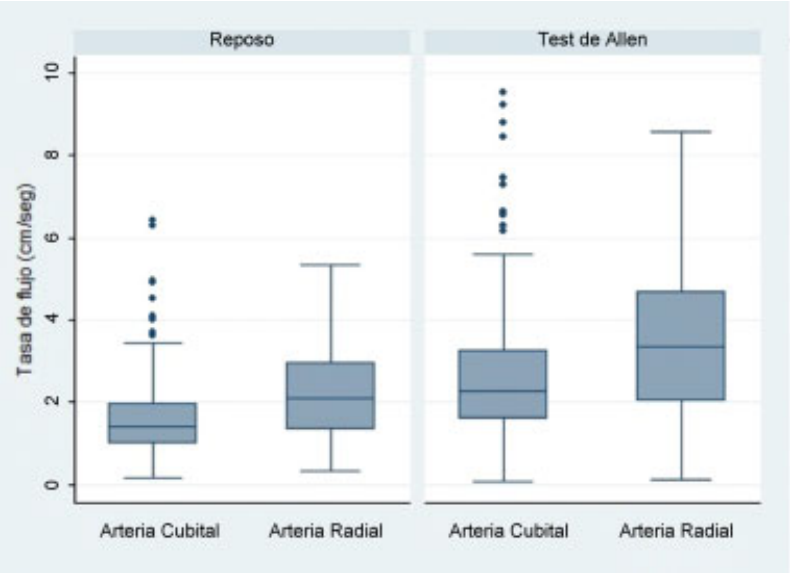

Fig. 7 Coeficientes de correlación para las tasas de flujo en reposo y con la prueba de Allen para las arterias radial y cubital.

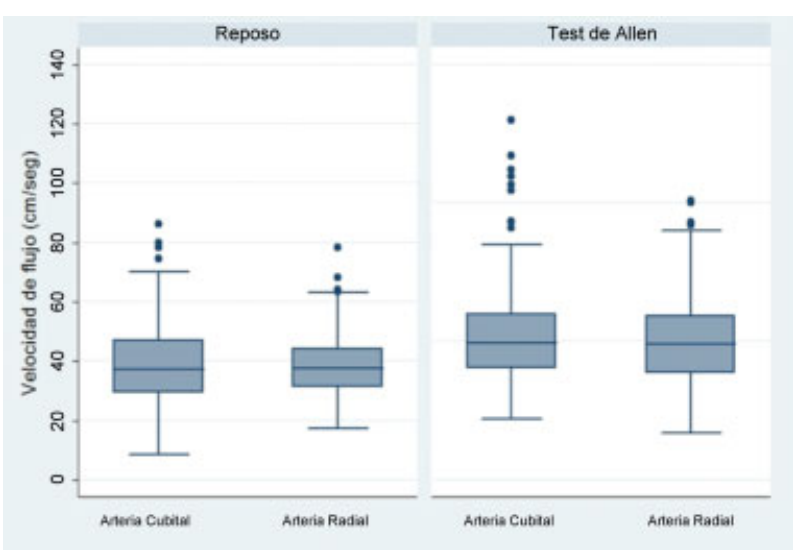

Fig. 8 Coeficientes de correlación para las velocidades de flujo en reposo y con la prueba de Allen para las arterias radial y cubital.

Es importante mencionar, como limitación principal del estudio, que la ecografía doppler color y la realización de la prueba de Allen, son herramientas "operador dependiente," sin embargo, las dos fueron realizadas por personal previamente entrenado para ello.

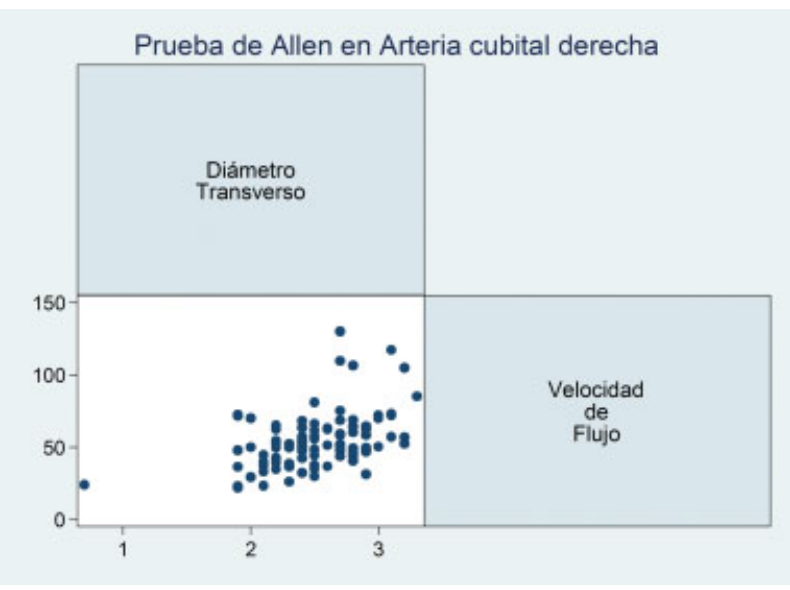

Fig. 10 Prueba de Allen en arteria cubital derecha. 


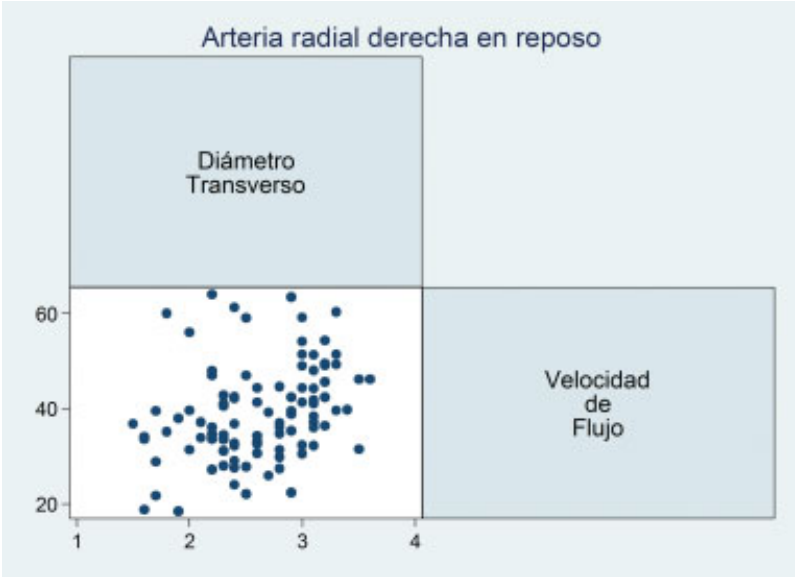

Fig. 11 Arteria radial derecha en reposo.

En cuanto a la influencia del tabaquismo, se ha documentado en estudios previos que el consumo de ése reduce el flujo sanguíneo a través de los grandes y pequeños vasos sanguíneos, ${ }^{3,12-14}$ sin embargo, es un factor que no fue tomado en cuenta en el presente estudio.

\section{Conclusión}

Es evidente que existen cambios adaptativos tanto anatómicos como funcionales en las arterias cubital y radial cuando hay ausencia de una u otra, lo que representa un sistema dinámico que se va a adaptar según el escenario presente.

Sin embargo, existe una clara dominancia de la arteria radial sobre la arteria cubital. Por la experiencia clínica dada por los procedimientos quirúrgicos realizados y por las lesiones traumáticas, esto no indica que la ausencia de este vaso comprometa la irrigación de las manos, siendo esto debido al sistema adaptativo de estas dos estructuras vasculares.

Conflictos de interese

Los autores no declaran ningún conflictos de interese.

\section{Bibliografía}

1 Clemente CD. A.B, Ph D, M.S, Dr. H.L. Sobotta atlas of human anatomy. 13 ed. Munich: Urban \& Fischer; 2001. 245 p.

2 OB.A morphometric study on the superficial palmar arch of the hand. In: Pinar YOM, Govsa F, editor. Surg Radiol Anat.; 2006. p.343-50.

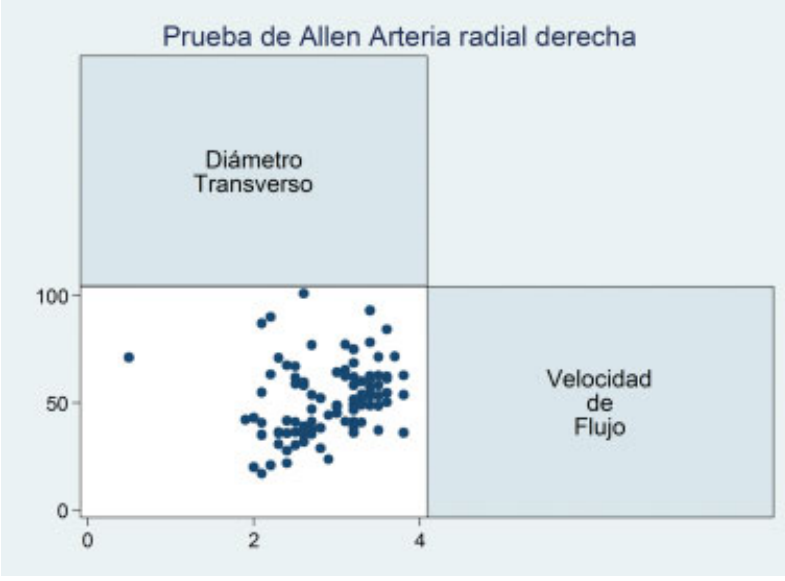

Fig. 12 Prueba de Allen en arteria radial derecha.

3 Ali SN, Srivastava S. Study of ulnar and radial arteries at wrist level in smokers. Scand J Plast Reconstr Surg Hand Surg 2008;42(06): 320-324

4 Kajikawa A, Ueda K, Mochizuki Y, Katsuragi Y. An objective Allen test using color Doppler echo. Plast Reconstr Surg 2010;125(02): 82e-85e

5 Doscher W, Viswanathan B, Stein T, Margolis IB. Hemodynamic assessment of the circulation in 200 normal hands. Ann Surg 1983;198(06):776-779

6 Tonks AM, Lawrence J, Lovie MJ. Comparison of ulnar and radial arterial blood-flow at the wrist. J Hand Surg [Br] 1995;20(02): 240-242

7 Haerle M, Häfner HM, Dietz K, Schaller HE, Brunelli F. Vascular dominance in the forearm. Plast Reconstr Surg 2003;111(06): 1891-1898

8 Cable DG, Mullany CJ, Schaff HV. The Allen test. Ann Thorac Surg 1999;67(03):876-877

9 Trager S, Pignataro M, Anderson J, Kleinert JM. Color flow Doppler: imaging the upper extremity. J Hand Surg Am 1993;18(04): 621-625

10 Krebs CA. Doppler Color. Giyanani VL, editor: Marban Libros; 2001. faltan el título del capítulo y las páginas

11 E. Tugsell P, Y. Asikoglu Z. Can forearm flaps be selected by use of color-flow duplex Doppler scanning? Eur J Plast Surg 1997; 20(06):297-299

12 van Adrichem LN, Hovius SE, van Strik R, van der Meulen JC. Acute effects of cigarette smoking on microcirculation of the thumb. $\mathrm{Br}$ J Plast Surg 1992;45(01):9-11

13 van Adrichem LN, Hovius SE, van Strik R, van der Meulen JC. The acute effect of cigarette smoking on the microcirculation of a replanted digit. J Hand Surg Am 1992;17(02):230-234

14 Ijzerman RG, Serne EH, van Weissenbruch MM, de Jongh RT, Stehouwer CD. Cigarette smoking is associated with an acute impairment of microvascular function in humans. Clin Sci (Lond) 2003;104(03):247-252 